\title{
ARCHIV DER PHARVIACIE.
}

CIV. Bandes zweites Heft.

\section{Erste Abtheilung.}

\section{Physik, Chemie und praktische Pharmacie.}

Versuch einer Lösung der Preisaufgabe über die Frage:

a) Wie viel reinen trocknen Succus Liquiritiae liefern die im Handel vorkommenden rohen Sorten des Lakritzens mit Allgabe des Preises und Stempels?

b) Wie verhält sich das aus den trocknen Wurzeln durch Auskochen, Verdunsten und starkes Einkochen erhaltene Extract im Vergleiche zu dem aus frischen Wurzeln dargestellten Succus?

Von

Albrecht Overbeck aus Lemgo ${ }^{*}$ ).

Motto: Coraggio sempre, il mio ragazzo! senza questa condizione non v'é riuscita. Coraggio per proseguire in tulti gli sludii onorevali. Silvio Pellico.

$\boldsymbol{Z}_{\mathrm{u}}$ der Arbeit wurden 6 verschiedene Sorten Lakritzen verwendet.

Erster Theil. Wie viel trocknen Lakritzen liefern die rohen Sorten:

1) Succ. liquirit. Bayonne mit dem Prädicat dünnstängig, nicht gestempelt, in vier Zoll langen Stangen von der

*) Die Arbeit ist von dem Verfasser vollkommen ohne alle Beihülfe ausgeführt, was hier aus guten Gründen angeführt wird. Sie wird hier in Auszuge mitgetheilt, in Folge eines Wunsches der Prüfungs-Commission, dem die Redaction gern nachgekommen ist.

Dr. Bley.

Arch. d. Pharm. CIV, Bds, 2. Hft. 
Dicke eines kleinen Fingers. Schon durch das äussere Aussehen gab sich diese Sorte als die schlechteste unter den sechs zu erkennen. Der Centner war mit $26 \frac{1}{2}$ Thlr. berechnet, also 1 Pfd. 7 Sgr. 245 Pf.

2) Succ. liquirit. calabric mit der Marke D. Langusso, 6-7 Zoll lange, meist viereckige, spröde und leicht zerbrechliche, auf dem Bruch stark glänzende Stangen. Der Centner kostet 33! Thlr., also das Pfund 9 Sgr. $1 \frac{2}{5}$ Pf.

3) Succ. liquirit. calabr. mit der Marke S. Raft. Von gleichen Eigenschaften, wie die vorhergehende Sorte, nur dass die Stangen nicht viereckig, sondern mehr rundlich, nach der Unterfläche aber platt gelegen sind. Der Centner kostet 33 Thlr., das Pfund 9 Sgr.

4) Succ. liquirit. von Abruzzo mit der Marke DV. Einen halben Fuss lange Stangen, dem Bayonner von Ansehen ähnlich, jedoch schöner. Das Pfund kostet 9 Sgr.

5) Succ. liquirit. calabr. mit der Marke R. de Rosa. Dicke Stangen von der Länge eines halben Fusses und darüber. Das Pfund kostet $11 \mathrm{Sgr}$.

6) Succ. liquirit. calabr. mit der Marke Duca di Corigliano. Der längste, dickste und schönste von allen, äusserlich wie auf dem Bruch stark glänzend, am gestempelten Ende verbreitert, durchaus hart und fest. Das Pfund kostet $12 \mathrm{Sgr}$.

Was nun die Darstellung des reinen Succus aus diesen rohen Sorten des Lakritzens betrifft, so wählte ich die schon längst von den tüchtigsten Pharmaceuten empfohlene, in neuester Zeit so bewährt gefundene nnd auch in der neuesten Preussischen Pharmakopöe sanctionirte Vorschrift.

1) S. Raft. Demgemäss schichtete ich in einem gläsernen Cylinder, welcher drei Finger hoch über dem Boden eine mit einem Korkstöpsel verschlossene Oeffnung hat, 100 Drachmen $=12 \frac{1}{2}$ Unzen des in Zoll lange Stuicken zerbrochenen S. Raft mit Stroh, so dass zu unterst vom Boden bis über die Oeffnung hinauf zuerst eine Lage Stroh war. Hierauf wurde so viel kaltes Wasser gegossen, dass die obersten Stuicken eben davon bedeckt waren. 
Nach vierundzwanzigstündigem ruhigem Stehen lüftete ich den Korkstöpsel mit der Vorsicht, dass die Flüssigkeit nur in einem ganz feinen Strahl ablief, sie war ziemlich dick, aber ganz klar. Als ich dieselbe Operation noch zweimal wiederholt hatte, war der rohe Succus ganz erschöpft. So sicher und so leicht führt gewiss keine andere Darstellungsweise des gereinigten Lakritzens zum Ziele. Die vereinten Fluissigkeiten wurden weiterhin in Dampfbade bis zur starken Extractconsistenz verdampft, und dann in kleinen Portionen in Papierkapseln vertheilt, in der Trockenstube soweit ausgetrocknet, dass sie sich zu Pulver zerreiben liessen. So erhielt ich vom Succ. liquirit. calabr. S. Raft 67 Proc. reinen trocknen Succus. Auf gleiche Weise behandelt, gab:

2) D. Langusso...... 72 Proc.

3) Duca di Corigliano. 8 80,5 》

4) Bayonne........ 51 》

5) Abruzzo.........6\% 60

6) R. de Rosa....... 78 » reinen trocknen Succ. liquirit.

b) Wie verhält sich das aus den Wurzeln durch Auskochung, Verdampfen und starkes Einkochen erhaltene Extract im Vergleich zu dem vorstehenden aus frischen Wurzeln dargestellten Succus?

2 Pfund p. c. gute, feste, stark gelbe Wurzeln der Glycyrrhiza glabra L., wovon der Centner mit 7 Thlr., das Pfund mit $2 \frac{1}{2} \mathrm{Sgr}$. nolirt waren, wurden sehr fein zerschnitten, mit der erforderlichen Menge Wasser im verzinnten kupfernen Kessel uber freiem Feuer gekocht und dann ausgepresst. Diese Operation wiederholte ich dreimal. Nach dem dritten Kochen und Auspressen waren sie dem Geschmack nach sehr gut erschöpft. Die vercinten Flüssigkeiten stellte ich in einem hohen Steintopfe 24. Stunden hindurch zum Absetzen bei Seite und klärte sie dann durch Decantiren und Coliren. Auf dem Colatario blieb eine weisse Masse zurück, die sich durch ihre starke Reaction auf Jodtinctur als ganz oder doch theilweise aus Amylum bestehend zu erkennen gab. Die klare Brühe 
wurde stark eingekocht, wobei sie sich wieder bedeutend trubte. Als sie bis aufs Drittel reducirt war, liess ich sie die Nacht hindurch wiederum absetzen und klärte sie am folgenden Morgen abermals durch Decantiren und Filtriren. Auf dem Filter blieb wieder eine Masse zurück, in der Jodtinctur das Amylum untrüglich nachwies. In einem flachen Kessel wurde nun die geklärte Flüssigkeit unter gegen das Ende hin unausgesetztem Rühren (jedoch so, dass das Wallen und Sieden keinen Augenblick unterbrochen wurde) stark eingekocht, bis zu dicker Extractconsistenz. Es wurden 10 Unzen Extract. spiss. erhalten.

Ferner wurde ein Theil davon noch weiter eingekocht, bis zur Pillenconsistenz ausgerollt und in gelinder Wärme ausgetrocknet.

Eine dritte Portion wurde in einem Porcellanschälchen zum völligen Austrocknen hingestellt, nach demselben zu Pulver zerrieben, und dieses in einem wohlverkorkten Glase aufbewahrt. \& Theile Extr. spiss. gaben 3 Theile Extr. sicc. Demnach war die Ausbeute von den beiden in Arbeit genommenen Pfunden Wurzeln $7 \frac{1}{2}$ Unzen Extr. sicc. oder, was dasselbe ist, 23,4 Proc.

So hatte ich nun, der Aufgabe gemäss, das Extr. liquirit. in dreierlei Form vor mir:

1) als Extr. spiss.

2) „Extr. in baculis.

3) „Extr. sicc. pulv.

Auc'l der Succus wurde in diese 3 Formen gebracht.

Zugleich muss ich hier noch bemerken, dass ich zu allen im Folgenden angegebenen vergleichenden Versuchen mit Extract und Succ. liquirit. da, wo es nicht näher angegeben ist, mich allemal des gereinigten Dúca di Corigliano bedient habe.

\section{A. Physische Eigenschaften.}

Die F arbe beider, des Extracts und des Succus, ist in allen drei Formen wesentlich von einander verschieden. Am stärksten tritt dieser Unterschied beim Pulver hervor. Das Pulver des Extracts nämlich ist hellzimmtfarben, der 
gepulverte Succus dagegen schwarzbaun. Im eingedickten Zustande sieht das Extract in Masse anfangs hellbraun aus, wird aber allmälig dunkler; der Succus dagegen ist von Anfang an ganz schwarz. Streicht man etwas davon auf Papier aus, so ist diese Farbenverschiedenheit wiederum sehr auffallend: das Extract erscheint auf diese Weise ockerfarhen, der Succus pechschwarz glänzend. -

In Stangenform ist das Extract anfangs auch sehr hell, dunkelt aber allmälig nach, und ist zuletzt vom Succus äusserlich nicht zu unterscheiden.

Der Geruch des Succus ist der bekannte eigenthümliche. Beim Extract tritt dieser specifische Geruch, wenn gleich stark, doch nicht in solchem Maasse hervor.

Die Verschiedenheil beider im Geschmack ist nicht gering; das Extract besitzt den specifischen widerlich süssen Geschmack im höchsten Grade, und kratzt hintennach stark im Schlunde, der Succus schmeckt bei weitem nicht so stark süss, kratzt gleichfalls und ist zugleich etwas bitterlich.

Sowohl das Extr. spiss, wie der Succ. inspiss., lösen sich in der hinreichenden Menge Wassers völlig klar auf.

Die Extractlösung wurde bald häutig, und es bildete sich ein reichlicher weisser Bodensatz, bei der Succuslösung trat die Schimmelbildung später, aber dann auch weit stärker ein, der Bodensatz dagegen war nicht so reichlich, hatte aber eine weit dunklere Farbe.

In Stangenform löst sich weder der Succus, noch das Extract klar auf. Durch viel Wasser wird zwar der Rückstand noch etwas vermindert, immer bleibt aber ein solcher.

Dasselbe Verhalten zeigen beide im trocknen gepulverten Zustande, wo die Menge des im Wasser unlöslichen Rückstandes noch beträchtlicher ist. Die von diesem durchs Filter getrennte klare Flüssigkeit giebt, nach dem völligen Austrocknen, beim Wiederauflösen in Wasser abermals einen Rückstand. Hieraus scheint doch hervorzugehen, dass es unmöglich ist, einen völlig klar auflöslichen Succus oder Extr. liquiv. in Pulverform zu bekommen. Um 
so mehr muss man sich wundern, dass die neueste Preuss. Pharmakopöe dennoch nur diesen trocknen gepulverten Succus vorschreibt, während der meiste Lakritzensaft in der Receptur doch im aufgelösten Zustande verordnet wird und in diesem Falle klare Auflöslichkeit nur gewünscht werden kann. Deshalb dürfte es weit zweckgemässer sein, wenn die Pharmakopöe ausser ihrem Succ. liquir. in Pulverform auch einen Succus inspiss. auffuhrte, der sich in verschlossenen Gefässen sehr gut hält, und wovon man den mehr oder minder grossen Gebrauch grössere oder kleinere Quantitäten Solution zum raschen Gebrauch anfertigen könnte. Anders, wie mit der vorstehend beschriebenen Auflöslichkeit des Succus liquirit. dep., verhält es sich mit Uer Auflöslichkeit des rohen Lakritzens. Oben ist gesagt, dass die völlig erschöpften Stangen des rohen Succus unverändert in Form und Gestalt im Cylinder zurückbleiben. Um mich über ihre Natur aufzuklären, kochte ich zuvörderst einen Theil davon mit Wasser, und decantirte dann von dem ungelösten, schwarzen, glänzenden Pulver. Durch Zusatz von Alkohol bekam es sogleich ein Ansehen, wie Geronnenes, welches sich später als ein käsig flockiger Niederschlag ausschied. Dieser bestand aus Amylum.

2). Das oben erwähnte, beim Kochen erhaltene, in kaltem wie in heissem Wasser unlösliche, schwarze, glänzende Pulver löste sich eben so wenig in kochendem Alkohol. Kalilauge dagegen löste schon in der Kälte soviel davon auf, dass die Fluissigkeit gelb wurde, beim Kochen damit wurde noch mehr gelöst, und die Flüssigkeit färbte sich dunkelbraun. Demnach kann ich es für weiter nichts als für Absatz oder oxydirten Extractivstoff halten (vielleicht ganz oder doch theilweise verändertes und somit unlöslich gewordenes Glycyrrhizin?).

Der ganze noch ubrige Theil der extrahirten Stangen des rohen Succus wurde geschlämmt. Auf diese Weise fanden sich zu unterst einige linsengrosse Kupferspänchen. Untersuchungen mit den ubrigen fünf Sorten des rohen Succus gaben dieselben Resultate. Bei allen bestand der 
Rückstand aus Amylum, oxydirtem Extractivstoff und metallischem Kupfer und zwar machte ersteres stets die Hauptmasse aus. Metallisches Kupfer fand sich bei allen verhältnissmässig nur sehr wenig vor.

Verhalten in der Wärme.

Das Extract war in weit kürzerer Zeit ausgetrocknet als der Succus bei der Wärme des Trockenofens.

Verhalten in feuchter Luft.

Beide zu gleicher Zeit feuchter Kellerluft ausgesetzt, zeigte sich der Succus längst zerflossen, als das Extract erst anfing weich zu werden.

Ferner bemerkte ich, dass sich weder der reine Succus, noch das selbstbereitete Extract, in Stangenform in gewöhnlicher Zimmerluft hielt, während sich doch der rohe Succus sehr gut hält. Wenn wir zurückblicken und sehen, dass der in kattem Wasser unlösliche Theil des rohen Lakritzens, der Hauptmasse nach, aus Amylum besteht, so haben wir hierin das Agens dieses verschiedenen Verhaltens des rohen und gereinigten Succus.

Wie aber dieses Amylum bineingekommen, ob es ursprünglich schon in der Wurzel gewesen oder dem Safte durch Verfälschung zugemischt sei, das ist eine andere Frage. Der Umstand, dass wir das Amylum in seinem gewöhnlichen Zustande, und nicht kleisterhaft darin vorfinden, scheint für die erste Ansicht zu sprechen. Da aber die Menge des Amylums, welche sich bei Bereitung des Extracts auf dem Filter und im Bodensatze fand, durchaus in keinem Verhältniss zu der Menge des im rohen Succus gefundenen steht, indem letztere vielfach grösser ist, so kann ich nicht anders als glauben, dass das Amylum dem rohen Succus um ihm mehr Haltbarkeit zu geben, oder auch, um sein Gewicht zu vermehren, falschlich zugemischt sei.

Demnach steht fest: die Haltbarkeit des rohen Succus beruht auf diesem Amylumgehalt und umgekehrt die Nichthaltbarkeit des durch Erschöpfen des rohen Lakritzens 
mit kaltem Wasser bereiteten reinen Succus eben auf diesem Mangel an Amylum.

Diesem Uebelstande der Nichthaltbarkeit und dem leichten Zerfliessen des gereinigten Lakritzens abzuhelfen, sind mancherlei Vorschläge gemacht.

So räth Dr. Geiseler, den rohen Lakritzen mit heissem Wasser auszuziehen, auf welche Weise ein sehr haltbarer Succ. liquirit. in bacillis erhalten werde.

Später schlug F orcke vor, dem durch Ausziehen des rohen Succus mit kaltem Wasser gewonnenen reinen Safte durch Zusatz von weissem Zuckerpulver (1-2 Theile Sach. alb. auf 16 Theile Succus) eine grössere Haltbarkeit zu ertheilen. Durch meine Versuche hat sich jedoch die Unzulänglichkeit dieser Methode erwiesen und glaube im Zusatz von $\frac{1}{16}$ Milchzucker den Stoff gefunden zu haben, den reinen Lakritzen haltbarer zu machen.

\section{B. Chemisches Verhalten.}

Der Kupfergehalt des Lakritzensaftes ist ein Hauptpunct, den wir näher ins Auge fassen müssen.

Hier soll erörtert werden, ob der rohe Succus sowohl, wie der daraus bereitete reine, Kupfer in aufgelöstem Zustande d.h. als Oxyd in Verbindung mit Säuren oder als Salz enthalte, und ob, wenn dies der Fall, dasselbe in gleicher Weise in selbstbereitetem Extracte sich finde.

Dem zufolge wurden zunächst von den 6 Sorten des rohen Lakritzens je 2 Drachmen in Wasser gelöst und mit einer blanken Messerklinge 12 Stunden lang geprüt. So fand sich in keinem Kupfer.

Ferner wurde in die concentrirten Lösungen des gereinigten Lakritzens und des Extracts, welche beide sauer reagirten, je eine polirte Stange gehangen. Nach Verlauf eines halben Tages *) zeigten dieselben nicht den geringsten rothen Anflug.

Sodann wurde eine Unze trockner Succ. depur. Cori-

*) Der Versuch hätte eine längere Zeit hindurch dauern müssen, wenn so ein Resultat erzielt werden sollte.

B. 
gliano in Schmelztiegel verkohlt. Die Kohle zerrieb ich in einem Porcellanmörser und breitete sie dann an der Wandung des nun schräg eingesetzten Tiegels aus und verwandelte dieselbe durch lebhaftes Feuer in Asche, übergoss solche nach dem Erkalten mit Salzsäure und digerirte das Gemisch bei gelinder Wärme. Alsdann wurde mit Wasser verdünnt und filtrirt; das (von Eisen) gelblich gefärbte Filtrat aber so lange mit Aetzammoniak versetzt, bis alles Eisen und alle Talk - und Kalkerdesalze vollständig gefällt waren. Die nun entstandene wasserhelle Flüssigkeit wurde filtrirt, mit Salzsäure neutralisirt und gab:

a) mit Schwefelwasserstoffwasser versetzt, nach mehrstündiger Ruhe keinerlei Färbung,

b) durch Kaliumeisencyanür nach längerem Stehen keine Reaction auf Kupfer und

c) war an einer polirten Nadel nach langer Zeit kein rother Beschlag, keine Reduction von Kupfer zu bemerken.

Auf gleiche Weise wurde 1 Unze des Extracts auf Kupfer geprüft, die Erscheinungen, sô wie auch das Resultat, waren die nämlichen.

Wenn nun auf die eine und auf die andere Weise, weder im rohen, noch im gereinigten Succus, noch im Extract eine Spur aufgelösten Kupfers gefunden werden konnte, so musste ich schon, zumal in Berücksichtigung des Umstandes, dass Kupfersalze (wie iiberhaupt alle Metallsalze) in den Lösungen des Succus und Extract. liquirit. Niederschläge hervorbingen, auf den Gedanken geführt werden, ob uberhaupt im Suissholzsafte Kupfer in aufgelöster Form vorkommen könne? Dies veranlasste mich, folgende Versuche anzustellen: 2 Drachmen Succus, desgl. 2 Drachmen Extract wurden in destillirtem Wasser gelöst, in je 4 Probirgläser vertheilt, digerirt

a) mit Cupr. raspal. grj,

b) " " oxyd. nigr. grj $\beta$,

c) " " sulphur. grjj.

d) " " acetic. grjj.

In allen 4 Fällen entstanden Niederschläge, von denen abfiltrirt wurde. Die Filtrate, mit einer polirten Nadel 
gepruft, gaben durchaus kein Kupfer zu erkennen, welches sofort zu erkennen war, als man geringe Spuren von Kupfersalzen zusetzte.

Das abweichende Verhalten des Succus vom Extract hierbei, dass nämlich letzteres mehr Kupfer fällte, als gleich viel Succus, müssen wir in dem Vorhandensein der grösseren oder geringeren Menge eines in beiden vorkommenden Körpers suchen. Dieser Körper ist der Süssholzzucker oder das Glycyrrhizin. So lange die Menge des Kupfers im Verhältniss zu der Menge des Glycyrrhizins nicht zu gross ist, kann im Suissholzsafte durchaus kein aufgelöstes Kupfer gefunden werden, da es selbst, wenn es anfangs aufgelöst sein sollte, mit dem Süssholzzucker alsbald eine unlösliche Verbindung eingeht. Hieraus ergiebt sich ferner der Schluss : dass in einem sorgfälig bereiteten Succ. dep. eben so wenig, wie im Extract überhaupt Kupfer vorkommen kann.

Um das weitere chemische Verhalten des Extracts zum Succus zu erforschen, wurde eine halbe Unze Extract, eben so viel Succus in je 8 Unzen destillirten Wassers gelöst und nun beide mit den verschiedenen Reagentien geprüft. Alle bei der Prüfung angewandten Salze, nämlich: essigsaures, salpetersaures, kohlensaures, neutrales weinsaures und saures weinsaures Kali, schwefelsaures Natron, schwefelsaure Magnesia, Chlorammonium, Boraxweinstein, Brechweinstein und Natronweinstein, brachten im Laufe des ersten Tages durchaus keine Veränderung, weder in den Lösungen des Extracts, noch des Succus hervor. Aber nach mehrern Tagen hatten sich in allen mehr oder minder grosse Niederschläge gebildet.

Blicken wir zurück, um die Natur der durch die verschiedenen Reagentien hervorgebrachten Niederschläge in etwas näher kennen zu lernen, so wissen wir zunächst, dass die durch Säuren und Metallsalze in den Lösungen, sowohl des Succus, als des Extracts, erzeugten, ihre Entstehung dem Vorhandensein des Glycyrrhizins zu verdanken haben und wirkliche Verbindungen eben dieses Glycyrrhizins mit den Säuren und Salzen oder Oxyden der entsprechenden Salze sind. Dies giebt uns den Wink, dass weder Säu- 
ren, noch Metallsalze, weder mit Extract, noch mit Succ. liquir. verordnet werden dürfen, wenn beide nicht in ihrer Wirkung bedeutend modificirt werden sollen. Dasselbe gilt von der Opiumtinctur, von schwefelsaurem Chinin und salpetersaurem Strychnin.

Um meine Vermuthung, dass die durch die oben angeführten Salze entstandenen Niederschläge, sowohl in der Succus- als Extracilösung, ihre Entstehung dem Glycyrrhizin zu verdanken haben möchten, gerechtfertigt zu sehen, stellte ich folgenden Versuch an:

100 Gran Succus, eben so viel Extract, löste ich in je 2 Unzen destillirten Wassers, und versetzle beide Lösungen mit Schwefelsäure im Ueberschuss. Alsdann filtrirte ich von den gebildeten Niederschlägen ab und erhielt so vom ersten 9 Gran, vom letzteren 24 Gran schwefelsaures Glycyrrhizin.

Ferner löste ich auf gleiche Weise 100 Gran Succus und 100 Gran Extract in je 2 Lnzen destillirten Wassers, und setzte zu jedem 100 Gran Chlorammonium. Nach mehreren Tagen war in beiden ein Niederschlag entstanden, von dem abfiltrirt und aus dem Filtrat durch Schwefelsäure das schwefelsaure Glycyrrhizin gefallt wurde. Vom Succus erhielt ich nun 5 Gran, vom Extract $13 \frac{1}{2}$ Gran schwefelsaures Glycyrrhizin.

Hieraus geht offenbar hervor, dass dic durch Salmiak in aufgelöstem Succus und Extract erzeugten Niederschläge glycyrrhizinhaltig sind, und deshalb die in solchen Mixturen sich bildenden Niederschläge keinesweges entfernt werden dürfen, da sie den wirksamsten Bestandtheil enthalten.

Der Umstand ferner, dass alle Reagentien in den Extractlösungen fast jedesmal bedeutendere Niederschläge und schneller hervorbrachten, als in den Lösungen des Succus, muss uns sogleich sehr auffallen und uns ermuntern, nach der Ursache dieser Erscheinung zu forschen. Wenn wir in Betracht ziehen, dass aus 100 Gran Succus 9 Gran, aus 100 Gran Extract dagegen 21 Gran schwefelsaures Glycyrrhizin durch überschüssige Schwefelsäure 
gefällt wurden, so werden wir auf den Gedanken gefuhrt, dass bei der grösseren Menge des Glycyrrhizins im Extract bedeutendere Niederschläge und schneller entstehen mussten, als bei der geringeren im Succus. Das Glycyrrhizin aber, oder, wie es Robiquet nannte, Glycion, ist eben der wichtigste Bestandtheil and bedingt wahrscheinlich dessen Wirksamkeit. Vom Gesichtspuncte der Ausgaben aus betrachtet, erschien es mir nothwendig. den Glycyrrhizingehalt des Extracts im Vergleich zu dem des Succus zu erforschen.

Zur Darstellung des Glycyrrhizins aus dem Süssholzsafte sind mehrere Vorschriften gegeben. Ich wählte die Berzelius'sche und erhielt, nach derselben bereitet, aus einer Unze Succus 33 Gran, aus einer Unze des selbst bereiteten Extracts dagegen auf gleiche Weise behandelt 73 Gran Glycyrrhizin.

Der Glycyrrhizingehalt des Extracts zu dem des Succus verhält sich also wie $73: 33$, ist also mehr denn das Doppelte so gross. Folglich besitzt auch das Extract eine mehr denn doppelt so grosse Wirksamkeit, als der Succus.

Wenn nun schon von verschiedenen Sciten her der Vorschlag gemacht ist, den Succus aus der Materia medica zu verbannen, und dafur das selbstbereitete Extract einzuführen: so muss es auch einem Jeden cinleuchten, wie wichtig es ist, ein minder wirksames Mittel durch ein mehr als doppelt so heilkräftiges zu verdrängen.

Fassen wir ferner die pecuniare Seite ins Auge, so verhält sich

$$
2 \frac{1}{2}: 23,4=12: 80, \ddot{3}
$$

d. h. der Preis des Extracts zu dem des Succus verhalt sich ungefahr wie $5: 6$.

Berücksichtigt man nun, dass das Extract eine mehr als doppelt so grosse Wirksamkeit besitzt, der Arzt demnach mit der Halfte Extract eben so vicl und mehr ausrichtet, als mit doppelt so vicl Succus, so kann man in Bezug hierauf mit Recht sagen, dass sich der Preis des 
Extracts zu dem Succus verhalte, wie bै:12; der Succus also noch mal so hoch zu stehen kommt, als das Extract, so dass ich nicht umhin kann, am Schlusse meiner kleinen Arbeit den Wunsch auszusprechen, dass das ungleich wirksamere und doch wohlfeilere Extract aus den trockenen Wurzeln recht bald den schlechteren, weit weniger wirksamen und dabei doch theurern Succus verdrängen möge. Und zwar könnte sich der Apotheker dasselbe am besten in dreierlei Form darstellen:

1) als trocknes Pulver zu Pillen und Pulvern.

2) als Stangen mit Milchzucker zum Handverkauf und

3) als dickes Extract, welches sich in wohl verschlossenen Gefässen sehr gut hält, und wovon man zu Zeit dem Bedarf entsprechende Mengen Solution zu Tropfen und Mixturen anfertigen würde.

Wenn diese meine Arbeit auch nur einigermaassen dazu beitragen sollte, den Gedanken, den Succ. liquir. baldmöglichst durch das Extrac. liquirit. zu verdrängen, zu fördern und endlich zu realisiren. so würde ich mich dadurch für meine Mühe hinreichend belohnt finden.

Verzeichniss der eingesandten Proben.

1) Duca di Corigliano depur. sicc. pulv. 2) R. de Rosa dep. non pulv. 3) S. Raft dep. sicc. pulv. 4) Abruzzo dep. non pulv. 5) Bayonne dep non pulv. 6) D. Langusso dep. sicc. pulv. 7) Extr. liquirit. sicc. pulv. 8) Corigliano dep. inspiss. 9) Extr. liquir. in bacillis. 10) Corigliano dep. in bacillis. 11) Extr. liquir. in bacillis cum Sacchar. alb. $\frac{1}{T-}$. 12) Corigliano in bacillis dep. cum Sacchar. alb. $\frac{1}{16}$. 13) Extr. liquir. in bacillis cum Sacchar. lactis $\frac{1}{16}$. 14) Corigliano dep. in bacillis cum Sacchar. lact. $\frac{1}{T}$. 15) Glycyrrhizin e succo liquir. Duca di Corigliano. 16) Glycyrrhizin ex extracto liquiritiae.

Die beigefuigten Proben haben durchaus allen Anforderungen der Preisaufgabe entsprochen. 
Diese Arbeit ist in der.General-Versammlung des Apotheker-Vereins am 13. September $1847 \mathrm{mit}$ dem ersten Preise belohnt worden.

\title{
Die Prüfungs - Commission.
}
Dr. L. F. Bley.
Dr. Geiseler.
Dr. L. As ch off.

\section{Ueber den frischen Seidensaft;}

\author{
von \\ Dr. Herm. Ludwig *).
}

In Jena's Umgegend findet sich hier und da der weisse Maulbeerbaum (Morus alba) angepflanzt; es sind junge kräftige Stämme, welche aus einer Zeit herstammen, wo von Preussen aus die Anregung zur Einführung der Seidenzucht in Deutschland gegeben wurde. In Thüringen blieb bis jetzt dieser Industriezweig in der Kindheit, für Jena jedoch wurde im vorigen Jahre der Beweis geliefert, dass hier so gut, wie in lialien und Frankreich mit Vortheil die schönste Scide gezogen werden könne, vorausgesetzt, dass die Seidenzucht rationell betrieben wird und Maulbeerblätter in hinreichender Menge zur Fütterung der Raupen vorhanden sind.

Herr v. Trentovius aus Memel, Mitglied des hiesi-

*) Die im Sommer des vorigen Jahres uns dargebotene Gelegenheit, die Seidenzucht in ausgezeichneter Weise ausgeführt zu sehen, veranlasste mich, Hrn. v. Trentovius um Mitheilung mehrerer, die Seidenzucht betreffender Gegenstände zur chemischen Untersuchung zubitten; denn es schien mir, als sei noch manche darauf bezügliche Frage durch die chemische Analyse zu erledigen. Hr. Dr. Ludwig, damals Assistent am hiesigen pharm.chemischen Institute, verfolgte dann den Gegenstand weiter. Die gewonnenen Resultate hat derselbe bereits in einem Vortrage bei der vorigjährigen General-Versammlung des norddeutschen A potheker-Vereins in Jena mitgetheilt. Dieselben werden in der gegenwärtigen Zusammenstellung anch unsern Lesern willlommen sein.

H. Wr. 